\title{
Circadian rhythm of cardiac troponin I and its clinical impact on the diagnostic accuracy for acute myocardial infarction
}

\author{
K. Wildi ${ }^{1}, 1^{*}$, H. Singeisen ${ }^{1 *}$, R. Twerenbold ${ }^{1}$, P. Badertscher ${ }^{1}$, D. Wussler ${ }^{1}$,
} L.J.J. Klinkenberg ${ }^{2}$, S.J.R. Meex², T. Nestelberger ${ }^{1}$, J. Boeddinghaus ${ }^{1}$, Ò. Miró $^{3}$, FJ Martin-Sanchez ${ }^{4}$, B. Morawiec ${ }^{5}$, P. Muzyk ${ }^{5}$, J. Parenica ${ }^{6}$, DI. Keller ${ }^{7}$, N. Geigy ${ }^{8}$, E. Potlukova ${ }^{1}$, Z. Sabti ${ }^{1}$, N. Kozhuharov ${ }^{1}$, C. Puelacher ${ }^{1}$, Jeanne du Fay de Lavallaz¹, M. Rubini Gimenez ${ }^{1}$, S. Shrestha', G. Marzano ${ }^{1}$, K. Rentsch $^{9}$, S. Osswald ${ }^{1}$, T. Reichlin ${ }^{1}$, C. Mueller ${ }^{1}$, for the APACE Investigators ${ }^{*}$

'Department of Cardiology and Cardiovascular Research Institute Basel (CRIB), University Hospital Basel, Basel, Switzerland. ${ }^{2}$ Department of Clinical Chemistry, Cardiovascular Research Institute Maastricht (CARIM), Maastricht University Medical Center (MUMC), Maastricht, the Netherlands. ${ }^{3}$ Emergency Department, Hospital Clinic, Barcelona, Catalonia, Spain; ${ }^{4}$ Servicio de Urgencias, Hospital Clínico San Carlos, Madrid, Spain; ${ }^{5} 2$ nd Department of Cardiology, School of Medicine with the Division of Dentistry in Zabrze, Medical University of Katowice, Poland; ${ }^{6}$ Department of Cardiology, University Hospital Brno, Brno, Czech Republic and Medical Faculty, Masaryk University, Brno, Czech Republic; ' ${ }^{7}$ Emergency Department, University Hospital Zurich, Switzerland; ${ }^{8}$ Emergency Department, Kantonsspital

Liestal, Switzerland; ${ }^{9}$ Department of Laboratory Medicine, University Hospital Basel, Switzerland; ${ }^{10}$ The Critical Care Research Group, The Prince Charles Hospital, Brisbane, Australia

All authors take responsibility for all aspects of the reliability and freedom from bias of the data presented and their discussed interpretation

*both authors have contributed equally and should be considered first author

\section{Short title: circadian rhythm of troponin I}

Word Count: $\mathbf{3 1 0 0}$

Correspondence to: Professor Christian Mueller, Department of Cardiology, University Hospital Basel, Petersgraben 4, CH-4031 Basel, Switzerland; Phone: + 416132865 49, Fax; +41 6126553 53; e-mail: Christian.Mueller@usb.ch

Clinical Trial Registration: NCT00470587, http://clinicaltrials.gov/show/NCT00470587 


\section{Disclosures, grant support and conflict of interest}

We disclose that Dr Mueller has received research grants from the Swiss National Science Foundation and the Swiss Heart Foundation, the European Union, the Cardiovascular Research Foundation Basel, 8sense, Abbott, ALERE, Brahms, Critical Diagnostics, Nanosphere, Roche, Siemens, and the University Hospital Basel, as well as speaker or consulting honoraria from Abbott, ALERE, Brahms, Cardiorentis, Novartis, Roche, and Siemens. Dr Meex has received non-financial support from Roche Diagnostics and Abbott Diagnostics. Dr Twerenbold has received grants from the Swiss National Science Foundation (P300PB-167803/1) and speaker honoraria/consulting honoraria from Roche, Abbott and Brahms. Dr Reichlin has received grants from the Swiss National Science Foundation (PASMP3-136995), the Swiss Heart Foundation, the University of Basel, the Professor Max Cloetta Foundation, the Department of Internal Medicine, University Hospital Basel and speakers honoraria from Brahms and Roche.

The authors designed the study, gathered and analysed the data, vouched for the data and analysis, wrote the paper and decided to publish. All authors fullfill the criteria for authorship and approved the final article.

Key Word List: acute myocardial infarction, diagnosis, high-sensitivity cardiac troponin I, circadian rhythm 


\section{List of abbreviations}

hs-cTnT: high-sensitivity cardiac troponin T

AMI: acute myocardial infarction

AUC: area under the curve

s-cTnl: sensitive cardiac troponin I

hs-cTnl: high-sensitivity cardiac troponin I

ECG: electrocardiography

APACE: Advantageous Predictors of Acute Coronary Syndrome Evaluation

ED: emergency department

STARD: Standard for Reporting of Diagnostic Accuracy Studies

eGFR: estimated glomerular filtration rate

MDRD: Modification of Diet in Renal Disease

SD: standard deviation

IQR: interquartile range 


\section{Abstract (250)}

Background: High-sensitivity cardiac troponin T (hs-cTnT) blood concentrations were shown to exhibit a diurnal rhythm, characterized by gradually decreasing concentrations throughout daytime, rising concentrations during nighttime and peak concentrations in the morning. We aimed to investigate whether this also applies to (h)s-cTnl assays and whether it would affect diagnostic accuracy for acute myocardial infarction (AMI).

Methods: Blood concentrations of cTnl were measured at presentation and after $1 \mathrm{~h}$ using four different cTnl assays: three commonly used sensitive (s-cTnl Architect, Ultra and Accu) and one experimental high-sensitivity assay (hs-cTnl Accu) in a prospective multicenter diagnostic study of patients presenting to the emergency department with suspected AMI. These concentrations and their diagnostic accuracy for AMI (quantified by the area under the curve (AUC)) were compared between morning (11 p.m. to 2 p.m.) and evening (2 p.m. to 11 p.m.) presenters.

Results: Among 2601 patients, AMI was the final diagnosis in $17.6 \%$ of patients. Concentrations of (h)s-cTnl as measured using all four assays were comparable in patients presenting in the morning versus patients presenting in the evening. Diagnostic accuracy for AMl of all four (h)s-cTnl assays were high and comparable between patients presenting in the morning versus presenting in the evening (AUC at presentation: 0.90 vs 0.93 for s-cTnl Architect; 0.91 vs 0.94 for s-cTnl Ultra; 0.89 vs 0.94 for s-cTnl Accu; 0.91 vs 0.94 for hs-cTnl Accu).

Conclusions: Cardiac Tnl does not seem to express a diurnal rhythm. Diagnostic accuracy for AMI is very high and does not differ with time of presentation. 


\section{Translational aspect of this work}

Hs-cTnT blood concentrations were shown to exhibit a diurnal rhythm, this has been proven to have no effect on diagnostic accuracy for AMI. Whether this also applies to (h)cTnl assays has not been investigated yet.

There was no difference in cTnl concentrations between morning and evening presenters in all diagnostic groups. The diagnostic accuracy for AMI was very high and similar in both time frames. These findings indicate that (h)s-cTnl expresses no circadian rhythm and is safe in use at all time of the day. 


\section{Introduction}

Acute myocardial infarction (AMI) is a major cause of cardiovascular death and disability worldwide [1]. Rapid identification of AMI is of paramount clinical importance for the timely initiation of effective treatment and based on three diagnostic cornerstones: clinical assessment, electrocardiography (ECG) and cardiac troponin (cTn) as a quantitative marker of cardiomyocyte injury [2].

The current clinical use of cTn is based on the assumption that cTn blood concentrations are similar at different times of the day allowing the use of standardized cut-off levels (e.g. the $99^{\text {th }}$ percentile) irrespective of the time of day [1-6]. The clinical introduction of high-sensitivity cTn (hs-cTn) assays, which allow the precise quantification of cTn even in the normal range, has enabled investigators to actually test this assumption. In fact, the dogma of random fluctuation around a homeostatic setpoint of $\mathrm{cTnT}$ and $\mathrm{cTnl}$ blood concentrations was recently challenged by the intriguing observation that hs-cTnT exhibited a diurnal rhythm in a small group of male subjects with type 2 diabetes [7]. The diurnal hs-cTnT rhythm was characterized by peak-levels during morning hours, a gradual decrease during the day with lower levels in the evening and increasing concentrations during nighttime. While the pathophysiological and evolutionary reasons for this phenomenon are still unknown, it could affect the early diagnosis of AMI in two ways: first, higher CTnT blood concentrations in the morning in non-AMI conditions would reduce the difference between cTnT in non-AMI versus AMI and thereby reduce diagnostic accuracy of cTnT blood concentrations; second, the rising pattern of cTnT due to a circadian rhythm in the night could reduce the diagnostic accuracy of cTnT changes from the first to the second blood draw, which complement absolute concentrations. A second pilot study could not find a circadian rhythm for cTnl when measured with two specific cTnl assays [8]. Given the poor standardization of cTnl 
assays and the enormous possible clinical consequences of such a circadian rhythm for cTnl, the most commonly used analyte in the early diagnosis of AMI worldwide, substantial uncertainty remains regarding this phenomenon.

We therefore aimed to address this important gap in knowledge by assessing differences in cTnl blood concentrations and their possible impact on the diagnostic accuracy for AMI between patients presenting in the morning versus those presenting in the evening hours using four (h)s-cTnl assays not previously investigated regarding circadian rhythm. 


\section{Methods}

\section{1 Study design}

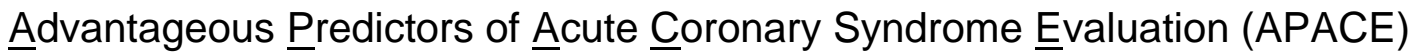

(http://clinicaltrials.gov/show/NCT00470587)_is an ongoing prospective diagnostic multicenter study enrolling patients aged at least 18 years presenting to the emergency department (ED) with symptoms suggestive for AMI and onset or peak of symptoms within 12 hours prior to presentation in twelve centers in five countries in Europe (Switzerland, Spain, Italy, Czech Republic, Poland) [9-11].

Patients were included irrespective of their renal function, only terminal kidney failure requiring regular dialysis was an exclusion criterion. For this analysis, patients were also excluded if A) time of presentation to the ED was missing, B) the investigated cTnl values were not available, C) the final diagnosis remained unclear after central adjudication and at least one hs-cTnT was elevated, thereby possibly indicating AMI and $\mathrm{D}$ ) patients had a final diagnosis of ST-elevation $\mathrm{MI}$, as cTn is considered of limited utility in the early diagnosis of these patients (Figure 1S, Online Suppl.). Patients were classified according to their time of presentation to the ED in two timeframes that were derived from the acrophase and bathyphase of the fitted cosine model of the observed diurnal rhythm of cTnT [7] to comprise the supposed highest and lowest levels of cTn: a cTn high-level phase in the morning (11:00 pm till 2:00 pm) and a cTn low-level phase in the evening $(2: 00 \mathrm{pm}$ till $11: 00 \mathrm{pm})[7,8]$. This strategy allowed us to make sure to include the supposed high and low of cTn as almost centering the respective time window and to allow enough patients in both time frames for a reasonable analysis since during nighttime less patients were included in relation to daytime.

For this study, blood sampling was performed at presentation to the ED (t1) as well as one hour thereafter (t2). 
The study complies to the principles of the Declaration of Helsinki and was approved by the local ethic committee. All patients provided written informed consent.

\subsection{Routine clinical assessment}

All patients underwent a clinical assessment that included medical history, physical examination, 12-lead ECG, continuous ECG monitoring, pulse oximetry, standard blood test, and chest radiography. Levels of the cTn used for the clinical management of patients (various assays, see online appendix) were measured at presentation and serially thereafter as long as clinically indicated. Treatment of patients was left at the discretion of the attending physician.

\subsection{Adjudicated diagnosis and follow-up}

Adjudication of the final diagnosis was performed centrally in a core lab (University Hospital Basel) for all patients. In order to take advantage of the higher sensitivity and higher overall diagnostic accuracy offered by high-sensitivity cTn (hs-cTn) [2-6,12,13], the adjudicating cardiologists had access to serial levels of cTn/hs-cTn measured locally as part of routine clinical care as well as serial hs-cTnT concentrations measured in a central laboratory from the serial study blood samples. Two independent cardiologists reviewed all available medical records - patient history, physical examination, results of laboratory testing, radiologic testing, ECG, echocardiography, cardiac exercise test, lesion severity and morphology in coronary angiography - pertaining to the patient from the time of ED presentation to 90-day follow up. In situations of disagreement about the diagnosis, cases were reviewed and discussed with a third cardiologist.

AMI was defined as recommended in current guidelines [2,14]. In brief, AMI was diagnosed when there was evidence of myocardial necrosis in a clinical setting consistent with myocardial ischemia. Necrosis was diagnosed by a rising or falling 
pattern of cTn with at least one value above the 99th percentile [2,15]. Unstable angina was diagnosed in patients with normal cTn levels and typical angina at rest, a deterioration of a previously stable angina, in cases of positive cardiac exercise testing or cardiac catheterization with coronary arteries found to have stenosis $\geq 70 \%$, and in ambiguous cases in which follow-up information revealed acute myocardial infarction or a sudden unexpected cardiac death within 90 days. Further predefined diagnostic categories included cardiac but not coronary symptoms (e.g., perimyocarditis, tachyarrhythmias, heart failure) and non-cardiac symptoms (e.g., muscular, pulmonary, gastrointestinal). If AMI was excluded in the ED but symptoms were highly suggestive of unstable angina, but no sufficient further work-up was performed to either reliably diagnose or exclude unstable angina, symptoms were classified as being of unknown origin. After hospital discharge, patients were followed at 3, 12 and 24 months by telephone or in written form, performed by trained researchers.

\subsection{Laboratory measurement}

Study blood samples were centrifuged and plasma/serum was frozen at $-80^{\circ} \mathrm{C}$ until cTnl concentrations were measured in a blinded fashion by a core laboratory team. For this study, three sensitive assays (s-cTnl Abbott Architect (9ng/L, 28ng/L, 14\% [16]), s-cTnl Siemens Ultra (6ng/L, 40ng/l, 8.8\% [16], s-cTnl Beckman Accu (10ng/L, 40ng/L, 14\% [16]) and one high-sensitivity assay (hs-cTnl Beckman Accu (2ng/L, 8.6ng/L, 10\% [16]) were used with the indicated limit of detection (LoD), $99^{\text {th }}$ percentile and coeffient of variation at the $99^{\text {th }}$ percentile respectively. The limit of blank (LoB) and LoD of hs-cTnT (Elecsys) were determined to be $3 \mathrm{ng} / \mathrm{l}$ and $5 \mathrm{ng} / \mathrm{l}$, the $99^{\text {th }}$ percentile of a healthy reference population was reported at $14 \mathrm{ng} / \mathrm{l}$ with an imprecision corresponding to $10 \%$ coefficient of variation (CV) at 13ng/l $[16,17]$.

The estimated glomerular filtration rate (eGFR) was calculated according to the modification of diet in renal disease study (MDRD) [18]. 


\subsection{Statistical analysis}

Categorical variables are reported as numbers and percentages, continuous Gaussian variables as means with standard deviation (SD), and non-Gaussian variables as medians with interquartile range (IQR). Comparisons between groups were made using Student`s t-test, Mann-Whitney U test and Kruskal-Wallis test as appropriate.

Absolute, unsigned change levels between $0 \mathrm{~h}$ and $1 \mathrm{~h}$ cTn were calculated (t2-t1). Median levels for $0 \mathrm{~h}, 1 \mathrm{~h}$ and absolute changes were compared in patients presenting in the morning versus evening group according to the five diagnostic groups. We hypothesized that if a circadian rhythm would be present for cTnl, it should become visible in patients with non-cardiac causes of chest discomfort and in patients with unstable angina, the two acute disorders in whom the cTnl concentration determined at presentation would be unchanged by the acute chest pain episode, but reflecting the "normal" circadian concentration.

Receiver operating characteristics curves (AUC) were constructed to assess sensitivity and specificity of cTnl for the diagnosis of AMI in morning and evening presenters. Comparison of independent AUC was performed as recommended by Hanley and McNeil [19]. Predefined subgroup analysis was performed in early presenters (defined as time from chest pain onset to presentation $\leq 3 \mathrm{~h}$ ) as a possible circadian rhythm in cTnl would be expected to particularly affect the diagnostic accuracy for AMI in this challenging subgroup.

All statistical analyses were performed using SPSS version 22 for Windows (Chicago, USA) and MedCalc 10.3.0 (Ostend, Belgium). All hypothesis testing was 2tailed and a significance level of less than 0.05 was considered statistically significant. 



\section{Results}

\subsection{Patient characteristics}

Between April 2006 and June 2013, a total of 2601 enrolled patients qualified for this analysis. Baseline values of s-cTnl Abbott Architect were available in 1266 patients (morning presenters 984 vs evening presenters 282 pts.), in 2567 patients for s-cTnl Siemens Ultra (1912 vs 655 pts.), in 1107 patients for s-cTnl Beckmann Accu (856 vs 251 pts.) and 1104 patients for hs-cTnl Beckman Accu (854 vs 250 pts.).

The adjudicated final diagnosis was AMI in $17.6 \%$ of patients, unstable angina in $10.1 \%$, cardiac non-coronary disease in $14.3 \%$, non-cardiac chest pain in $53.2 \%$ and unknown (but not $\mathrm{AMI}$ ) in $4.8 \%$ in the whole cohort. Overall, baseline characteristics in morning and evening presenters were comparable (Table 1), as were the baseline characteristics in the subgroups of patients with Oh values of the respective assays available (Table 1S, Online Supplement).

\subsection{Blood concentrations of cTnl}

Blood concentrations of $\mathrm{cTnl}$ at presentation, at $1 \mathrm{~h}$, and their absolute change at $1 \mathrm{~h}$ were comparable between morning versus evening presenting patients for all assays among the five diagnosis groups (Table 2S, Online Suppl.), also in patients adjudicated to have non-cardiac chest pain (Figure 1) and unstable angina.

\subsection{Impact on diagnostic accuracy for AMI}

For all assays, diagnostic accuracy for AMI as quantified by the AUC for cTnl concentrations at presentation was very high in morning and evening presenters: 0.90 vs 0.93 for s-cTnl Abbott Architect, 0.91 vs 0.94 for s-cTnl Siemens Ultra, 0.89 vs 0.94 for s-cTnl Beckman Accu and 0.91 vs 0.94 for hs-cTnl Beckman Accu ( $p$ for 
comparison=ns) (Table 2A, Figure 2). The AUC at $1 \mathrm{~h}$ and for the combination of absolute changes with baseline values was also very high and comparable among patients presenting in the morning versus patients presenting in the evening hours (Table 2A).

Among the subgroup of early presenting patients ( $\leq 3$ hours since symptom onset, $n=1401,53.9 \%$ ) similar results were observed (Table 2B). Nevertheless, there was one exception: the AUC at presentation for cTnI Siemens Ultra was significantly lower for patients presenting morning as compared to those presenting in the evening hours $(0.89$ $(95 \% \mathrm{Cl} 0.87-0.91)$ vs $0.95(95 \% \mathrm{Cl} 0.92-0.97))$.

\subsection{Prognostic accuracy of cTnl for the prediction of all-cause mortality}

As shown in Table 3S in the Online Supplement, there was no difference in accuracy regarding the prediction of all-cause mortality within 2 years between early-morning and evening presenters in all assessed (h)s-cTnl assays. 


\section{Discussion}

In a large diagnostic multicenter study enrolling patients presenting with suspected AMI to the ED we assessed the possible presence of a circadian rhythm of cTnl by using three sensitive and one high-sensitivity cTnl assay. We evaluated differences in cTnl blood concentrations and their possible impact on the diagnostic accuracy for AMI between patients presenting in the morning versus those presenting in the evening. We report three major findings:

First, cTnl blood concentrations at all time points as well as their absolute changes as measured with all four assays were similar in morning and evening presenters in all adjudicated diagnosis groups, particularly also in patients with noncardiac chest pain and unstable angina, the two diagnostic groups most likely to display a circadian rhythm. This finding strongly argues against the presence of a circadian rhythm for cTnl as quantified by the four cTnl assays tested. Second, diagnostic accuracy of all investigated assays for AMI was very high and comparable between patients presenting in the morning versus patients presenting in the evening. Third, similar results were reproducible in the subgroup of early presenting patients with the exception of s-cTnl Ultra at presentation.

These findings extend and corroborate previous pilot studies examining circadian rhythms of $c \operatorname{TnT}$ and $\mathrm{cTnl}[7,8]$ and further support the evolving concept that relevant pathophysiological differences underlie the release of $\mathrm{cTnT}$ and cTnl into the circulation, as well as possibly their clearance $[7,8,16,20]$. As the circulating $\mathrm{CTnT}$ and $\mathrm{CTnI}$ concentration is defined by the extent of release and clearance, this study does not allow to quantify the influence of both processes on the diurnal cTnT rhythm [8]. The rhythmicity observed for CTnT is in line with the circadian organization of the cardiovascular system [21]. Synchronizing physiological functions to the natural $24 \mathrm{~h}$ 
light-dark cycle, for example by upregulating body temperature and blood pressure before the time of awakening, anticipates to the demands of daytime activities [8]. Circadian rhythmicity has in addition been described for many cardiovascular traits: heart rate, sympathetic activity, vascular resistance, the renin-angiotensin-aldosteron system, prothrombotic tendency, platelet aggregability and the fibrinolytic system $[8,22-$ 24]. Identical to cTnT concentrations, all these components exhibit peak activity in (early) morning hours. The physiological rhythmicity of these cardiovascular components is reflected in the increasing intensity of cardiovascular events in the morning hours [25-27]. For example, the incidence of AMI, sudden cardiac death and stroke display a significant $24 \mathrm{~h}$ variation, characterized by a higher frequency in morning hours [25-27].

As the blood concentration of cTnl reflects the balance between release from cardiomyocytes into the systemic circulation and the clearance from blood, the absence of a circadian rhythm for cTnl in its blood concentration as documented by this study and a pilot study [7] does not necessarily prove the absence of a circadian rhythm in the release in cTnl. Assuming that the magnitude of clearance of cTnl from blood underlies a similar circadian pattern as its release from cardiomyocytes, then the net result could still be constant blood concentrations. The difference between cTnl and cTnT $[7,8]$ could therefore still be attributable to differences in their clearance mechanisms.

The clinical availability of hs-cTn assays for cTnT and cTnl has allowed translational and clinical research to provide first insights into differences between cTnT and cTnl [28-32]. Next to multiple and important similarities including a very high correlation in several clinical settings including suspected AMI in the ED [32], nearly exclusive organ specificity for the heart, very high diagnostic accuracy for AMI, and integral part of the universal definition of $\mathrm{MI}$ [2], recently observed differences include: first, higher prognostic accuracy for death for cTnT [32], higher prognostic accuracy for 
future Ml for cTnl [32], possible higher diagnostic accuracy in early presenters for cTnl [32], and circadian rhythm for cTnT but not cTnl [7,8].

The reasons for these differences are largely unknown. Both cTnT and cTnl modulate the contractile function of the sarcomere and therefore play an important role in the regulation of the excitation-contraction coupling in the heart [33]. However, they differ in regards of quantity of release into the bloodstream: cTnT content per gram of myocardium is roughly twice that of cTnl $(10 \mathrm{mg} / \mathrm{g}$ versus $4-6 \mathrm{mg} / \mathrm{g})$ [33]. Further, release kinetics differ: the part of cTn which occurs as a free cytosolic component (cTnT: 7\%, cTnl: $3.5 \%$ ) is released earlier into the blood stream in case of cellular degradation compared to the cTn fraction, which is bound in the sarcomere $[34,35]$. Additionally, differences in clearance of the two molecules might play a role: cTnT seems to have a higher molecular weight than cTnl (35kDa versus $23-24 \mathrm{kDa})$ [33]. It is yet thought that cTnT as well as cTnl are cleared by the kidney, but in patients with renal failure, cTnT is increased more frequently than cTnl [35]. It might therefore be possible that a diurnal change in cTnT clearance could contribute to the circadian variability described $[7,8,36]$ previously.

Moreover, specific characteristics of the individual cTn assays used might have contributed to the findings of this study. Besides the intraindividual biological variation, also interindividual variation as well as analytical variation, expressed by the coefficient of variation, must be considered when interpreting serially measured cTn values. As the hs-cTnl assay used in this study had even higher precision as the hs-cTnT assay used to detect the circadian rhythm for CTnT [7], this aspect does not seem to be responsible for the observed difference.

In the subgroup of early presenters, the AUC for the Oh concentrations of s-cTnl Ultra was lower for morning compared to evening presenting patients. Although this could have been attributable to a circadian rhythm with higher blood concentrations in non-AMI patients in the morning, it seems most likely a chance finding as no difference in absolute 
s-cTnl ultra blood concentrations were observed in non-AMI patients including patients with non-cardiac chest pain and patients with unstable angina at presentation and at $1 \mathrm{~h}$.

Scrutiny regarding possible confounders in blood concentrations of hs-cTnT and hs-cTnl is timely as several additional indications for clinical use beyond the detection of AMI have recently emerged, including the detection of exercise-induced myocardial ischemia and the quantification of myocardial end organ damage secondary to arterial hypertension or diabetes mellitus [37-39]. These indications have in common, that they rely on the ability to detect minuscule differences in cTnT and cTnl blood concentrations in the normal range. Presence of a circadian rhythm for cTnT or cTnl, as well as any other confounder would therefore be expected to have an even larger detrimental effect on these evolving indications as compared to the use of $\mathrm{cTnT}$ and $\mathrm{cTnl}$ in the detection of AMI $[7,8]$.

Several limitations of this study merit consideration. First, we evaluated patients presenting with acute chest discomfort to the ED. It is unknown whether our findings can be extrapolated to patients presenting to primary care. Second, we cannot comment on a circadian rhythm in patients with terminal kidney failure requiring dialysis, since these were excluded from our study. Third, the methodology of this study focuses on interindividual comparisons, as these are of great relevance when considering the current definition of $\mathrm{Ml}$, which uses as a mandatory criteria an elevation in cTn above a general cut-off level (the $99^{\text {th }}$ percentile). Additional studies focusing on intra-individual comparisons will provide additional insights.

In conclusion, in contrast to cTnT, cTnl does not seem to have a clinically relevant circadian rhythm. Diagnostic accuracy of cTnl is very high and does not differ significantly with time of presentation to the ED. 


\section{Other APACE Investigators ${ }^{*}$ and contributors to this manuscript include:}

Kathrin Meissner ${ }^{1}$, Caroline Kulangara ${ }^{1}$, Michael Freese ${ }^{1,3}$, Claudia Stelzig ${ }^{1}$, Roland Bingisser ${ }^{2,3}$, Christian Nickel², Beatriz López ${ }^{4}$, Esther Rodriguez-Adrada ${ }^{5}$, Damian Kawecki ${ }^{6}$, Eva Ganovská ${ }^{7}$, Jens Lohrmann ${ }^{1}$, Andreas Buser ${ }^{8}$, Dayana Flores ${ }^{1,3}$, Karin Grimm $^{1}$, Beate Hartmann ${ }^{1}$, Nicolas Schaerli ${ }^{1}$, Lorraine Sazgary ${ }^{1}$, Deborah Mueller ${ }^{1}$, Stella Marbot ${ }^{1}$.

${ }^{1}$ Cardiovascular Research Institute Basel (CRIB) and Department of Cardiology, University Hospital Basel, University of Basel; ${ }^{2}$ Department of Emergency Medicine, University Hospital Basel, University Basel, both Switzerland; ${ }^{3}$ GREAT network; ${ }^{4}$ Emergency Department, Hospital Clinic, Barcelona, Catalonia, Spain; ${ }^{5}$ Servicio de Urgencias, Hospital Clínico San Carlos, Madrid, Spain; ${ }^{6} 2$ nd Department of Cardiology, School of Medicine with the Division of Dentistry in Zabrze, Medical University of Katowice, Poland; ${ }^{7}$ Department of Cardiology, University Hospital Brno, Brno, Czech Republic and Medical Faculty, Masaryk University, Brno, Czech Republic; ${ }^{8}$ Blood Transfusion Centre, Swiss Red Cross, Basel, Switzerland and Department of Hematology, University Hospital Basel, University Basel, Switzerland.

\section{Acknowledgements}

We thank the patients who participated in the study, the staff of the emergency department, the laboratory technicians, and particularly Esther Garrido, Irina Klimmeck, Melanie Wieland, Kirsten Hochholzer, Ina Ferel, Janine Voegele and Fausta Chiaverio for their most valuable efforts. 


\section{References}

[1] S. Goodacre, E. Cross, J. Arnold, K. Angelini, S. Capewell, J. Nicholl, The health care burden of acute chest pain, Heart. 91 (2005) 229-230.

[2] K. Thygesen, J.S. Alpert, A.S. Jaffe, M.L. Simoons, B.R. Chaitman, H.D. White et al. Third universal definition of myocardial infarction, European Heart Journal. 33 (2012) 2551-2567.

[3] E. Giannitsis, H. Katus, Cardiac troponin level elevations not related to acute coronary syndromes, Nature Reviews Cardiology. 10 (2013) 623-634.

[4] R. Twerenbold, A. Jaffe, T. Reichlin, M. Reiter, C. Mueller, High-sensitive troponin T measurements: what do we gain and what are the challenges?, European Heart Journal. 33 (2012) 579-86.

[5] K. Thygesen, J. Mair, H. Katus, M. Plebani, P. Venge, P. Collinson et al, Recommendations for the use of cardiac troponin measurement in acute cardiac care., European Heart Journal. 31 (2010) 2197-204.

[6] T. Reichlin, A. Irfan, R. Twerenbold, M. Reiter, W. Hochholzer, H. Burkhalter et al, Utility of absolute and relative changes in cardiac troponin concentrations in the early diagnosis of acute myocardial infarction., Circulation. 124 (2011) 13645.

[7] L.J.J. Klinkenberg, J.-W. van Dijk, F.E.S. Tan, L.J.C. van Loon, M.P. van Dieijen-Visser, S.J.R. Meex, Circulating cardiac troponin T exhibits a diurnal rhythm, Journal of the American College of Cardiology. 63 (2014) 1788-1795.

[8] L.J.J. Klinkenberg, K. Wildi, N. van der Linden, I.W.K. Kouw, M. Niens, R. Twerenbold et al, Diurnal rhythm of cardiac troponin: consequences for the diagnosis of acute myocardial infarction, Clinical Chemistry. 62 (2016) 16021611.

[9] K. Wildi, L. Cullen, R. Twerenbold, J.H. Greenslade, W. Parsonage, J. Boeddinghaus et al, Direct comparison of 2 rule-out strategies for acute myocardial infarction: 2-h accelerated diagnostic protocol vs 2-h algorithm, Clinical Chemistry. 63 (2017) 1227-1236.

[10] R. Twerenbold, P. Badertscher, J. Boeddinghaus, T. Nestelberger, K. Wildi, M. Rubini Gimenez et al, Effect of the FDA Regulatory Approach on the 0/1-h Algorithm for Rapid Diagnosis of MI, Journal of the American College of Cardiology. 70 (2017) 1532-1534.

[11] R. Twerenbold, C. Jaeger, M. Rubini Gimenez, K. Wildi, T. Reichlin, T. Nestelberger et al, Impact of high-sensitivity cardiac troponin on use of coronary angiography, cardiac stress testing, and time to discharge in suspected acute myocardial infarction, European Heart Journal. 37 (2016) 3324-3332.

[12] J.A. de Lemos, Increasingly sensitive assays for cardiac troponins - a review, J Am Med Assoc. 309 (2013) 2262-2269.

[13] R. Twerenbold, T. Reichlina, M. Reiter, C. Mueller, High-sensitive cardiac troponin : friend or foe?, Swiss Medical Weekly. 141 (2011) 1-8.

[14] K. Thygesen, J.S. Alpert, H.D. White, A.S. Jaffe, F.S. Apple, M. Galvani et al, Universal definition of myocardial infarction, Eur Heart J. 28 (2007) 2525-2538.

[15] M. Roffi, C. Patrono, J. Collet, C. Mueller, M. Valgimigli, F. Andreotti et al, 2015 ESC Guidelines for the management of acute coronary syndromes in patients presenting without persistent ST- segment elevation, American College of Cardiology, European Heart Journal. 37 (2016) 267-315.

[16] F.S. Apple, Y. Sandoval, A.S. Jaffe, J. Ordonez-Llanos, IFCC Task Force on Clinical Applications of Cardiac Bio-Markers, Cardiac Troponin Assays: Guide to 
Understanding Analytical Characteristics and Their Impact on Clinical Care, Clinical Chemistry. 63 (2017) 73-81.

[17] E. Giannitsis, K. Kurz, K. Hallermayer, J. Jarausch, A.S. Jaffe, H. A. Katus, Analytical validation of a high-sensitivity cardiac troponin T assay, Clinical Chemistry. 56 (2010) 254-261.

[18] A.S. Levey, J.P. Bosch, J.B. Lewis, T. Greene, A more accurate method to estimate glomerular filtration rate from serum creatinine: a new prediction equation, Annals of Internal Medicine. 130 (1999) 461-470.

[19] E.R. DeLong, D.M. DeLong, D.L. Clarke-Pearson, Comparing the areas under two or more correlated receiver operating characteristic curves: a nonparametric approach, Biometrics. 44 (1988) 837-845.

[20] M. Roffi, C. Patrono, J.-P. Collet, C. Mueller, M. Valgimigli, F. Andreott et al, 2015 ESC Guidelines for the management of acute coronary syndromes in patients presenting without persistent ST-segment elevation, European Heart Journal. (2015) 267-315.

[21] M. Sato, T. Matsuo, H. Atmore, M. Akashi, Possible contribution of chronobiology to cardiovascular health, Frontiers in Physiology. 4 (2014) 409.

[22] G.H. Tofler, D. Brezinski, A.I. Schafer, C.A. Czeisler, J.D. Rutherford, S.N. Willich et al, Concurrent Morning Increase in Platelet Aggregability and the Risk of Myocardial Infarction and Sudden Cardiac Death, New England Journal of Medicine. 316 (1987) 1514-1518.

[23] G. Fearnley, G. Balmforth, E. Fearnley, Evidence of a diurnal fibrinolytic rhythm; with a simple method of measuring natural fibrinolysis., Clinical Science. 16 (1957) 645-50.

[24] J.A. Panza, S.E. Epstein, A.A. Quyyumi, Circadian Variation in Vascular Tone and Its Relation to a-Sympathetic Vasoconstrictor Activity, New England Journal of Medicine. 325 (1991) 986-990.

[25] R. Manfredini, B. Boari, R. Salmi, F. Fabbian, M. Pala, R. Tiseo, F. Portaluppi, Twenty-Four-Hour Patterns in Occurrence and Pathophysiology of Acute Cardiovascular Events and Ischemic Heart Disease, Chronobiology International. 30 (2013) 6-16.

[26] J.E. Muller, P.L. Ludmer, S.N. Willich, G.H. Tofler, G. Aylmer, I. Klangos et al, Circadian variation in the frequency of sudden cardiac death., Circulation. 75 (1987) 131-8.

[27] C. Argentino, D. Toni, M. Rasura, F. Violi, M.L. Sacchetti, A. Allegrett et al, Circadian variation in the frequency of ischemic stroke., Stroke. 21 (1990) 3879.

[28] T. Reichlin, A. Irfan, R. Twerenbold, M. Reiter, W. Hochholzer, H. Burkhalter et al, Utility of absolute and relative changes in cardiac troponin concentrations in the early diagnosis of acute myocardial infarction, Circulation. 124 (2011) 136145.

[29] G. Lippi, G. Targher, M. Franchini, M. Plebani, Genetic and biochemical heterogeneity of cardiac troponins: clinical and laboratory implications., Clinical Chemistry and Laboratory Medicine: CCLM/FESCC. 47 (2009) 1183-94.

[30] O. Bergmann, R.D. Bhardwaj, S. Bernard, S. Zdunek, F. Barnabé-, S. Walsh, J. Zupicic et al, Evidence for cardiomyocyte renewal in humans, Science. 324 (2009) 98-102.

[31] D. Needham, K. Shufelt, G. Tomlinson, J. Scholey, G. Newton, Troponin I and T levels in renal failure patients without acute coronary syndrome: a systematic review of the literature, Canadian Journal of Cardiology. 20 (2004) 1212-1218.

[32] M. Rubini Gimenez, R. Twerenbold, T. Reichlin, K. Wildi, P. Haaf, M. Schaefer et al, Direct comparison of high-sensitivity-cardiac troponin I vs. T for the early diagnosis of acute myocardial infarction., European Heart Journal. (2014) 1-9. 
[33] F.S. Apple, P.O. Collinson, Analytical characteristics of high-sensitivity cardiac troponin assays, Clinical Chemistry. 58 (2012) 54-61.

[34] B.J. Freda, W.H.W. Tang, F. Van Lente, W.F. Peacock, G.S. Francis, Cardiac troponins in renal insufficiency: Review and clinical implications, Journal of the American College of Cardiology. 40 (2002) 2065-2071. d

[35] R. Twerenbold, K. Wildi, C. Jaeger, M. Rubini Gimenez, M. Reiter, T. Reichlin et al, Optimal Cut-Off Levels of More Sensitive Cardiac Troponin Assays for the Early Diagnosis of Myocardial Infarction in Patients with Renal Dysfunction, Circulation. 131 (2015) 2041-50.

[36] N. van der Linden, J.M. Hilderink, T. Cornelis, D.M. Kimenai, L.J.J. Klinkenberg, W.P. van Doorn et al, Twenty-Four-Hour Biological Variation Profiles of Cardiac Troponin I in Individuals with or without Chronic Kidney Disease, Clinical Chemistry. (2017) clinchem.2017.275107.

[37] Y. Tanglay, R. Twerenbold, G. Lee, M. Wagener, U. Honegger, C. Puelacher et al, Incremental value of a single high-sensitivity cardiac troponin I measurement to rule out myocardial ischemia, The American Journal of Medicine. 128 (2015) 638-46.

[38] A. Jagodzinski, J.T. Neumann, F. Ojeda, N.A. Sörensen, P. Wild, T. Münzel et al, Cardiovascular Biomarkers in Hypertensive Patients with Medical TreatmentResults from the Randomized TEAMSTA Protect I Trial., Clinical Chemistry. (2017) clinchem.2017.275289.

[39] J.L. Januzzi, J. Butler, P. Jarolim, N. Sattar, U. Vijapurkar, M. Desai et al, Effects of Canagliflozin on Cardiovascular Biomarkers in Older Adults With Type 2 Diabetes, Journal of the American College of Cardiology. 70 (2017) 704-712. 


\section{Tables}

Table 1: Baseline characteristics of all patients

\begin{tabular}{|c|c|c|c|c|}
\hline & $\begin{array}{c}\text { all patients, } \\
\mathrm{n}=2601\end{array}$ & $\begin{array}{c}\text { morning presenters }{ }^{\mathrm{a}} \text {, } \\
\mathrm{n}=1935\end{array}$ & $\begin{array}{c}\text { evening presenters } \\
n=666\end{array}$ & $\begin{array}{l}p- \\
\text { value }\end{array}$ \\
\hline Age (years) & $62(49.0-74.0)$ & $62.0(50.0-75.0)$ & $59.5(48.0-72.0)$ & 0.01 \\
\hline Women, n (\%) & $822(31.6)$ & $613(31.7)$ & $209(31.4)$ & 0.4 \\
\hline Body-mass index $\left(\mathrm{kg} / \mathrm{m}^{2}\right)$ & $26.4(23.9-29.6)$ & $26.3(23.8-29.6)$ & $26.7(24.1-29.6)$ & 0.2 \\
\hline eGFR (ml/min/1.7) & $85.4(69.4-101.5)$ & $85.1(69.4-101.5)$ & $86(69.4-101.5)$ & 0.4 \\
\hline \multicolumn{5}{|l|}{ Risk factors, n (\%) } \\
\hline Hypertension & $1605(61.7)$ & $1205(62.3)$ & $400(60.1)$ & 0.3 \\
\hline Hypercholesterolemia & $1308(50.3)$ & $973(50.3)$ & $335(50.3)$ & 0.9 \\
\hline Diabetes mellitus & $443(17.0)$ & $337(17.4)$ & $106(15.9)$ & 0.4 \\
\hline current or previous smoking & $1619(62.2)$ & $1192(61.6)$ & $427(64.1)$ & 0.2 \\
\hline Family history & $690(26.5)$ & $502(25.9)$ & $188(28.2)$ & 0.2 \\
\hline \multicolumn{5}{|l|}{ History, n (\%) } \\
\hline Coronary artery disease & $905(34.8)$ & $682(35.2)$ & $223(33.5)$ & 0.4 \\
\hline previous AMI & $613(23.6)$ & $468(24.2)$ & $145(21.8)$ & 0.2 \\
\hline previous revascularization & $728(28.0)$ & $541(28.0)$ & $187(28.1)$ & 1.0 \\
\hline peripheral artery disease & $155(6.0)$ & $113(5.8)$ & $42(6.3)$ & 0.7 \\
\hline previous stroke & $142(5.5)$ & $110(5.7)$ & $32(4.8)$ & 0.4 \\
\hline \multicolumn{5}{|l|}{ ECG findings, $n(\%)$} \\
\hline Left bundle branch block & $26(1.0)$ & $19(1.0)$ & $7(1.1)$ & 0.9 \\
\hline ST-segment depression & $263(10.1)$ & $193(10.0)$ & $70(10.5)$ & 0.7 \\
\hline T-wave inversion & $334(12.8)$ & $240(12.4)$ & $94(14.1)$ & 0.3 \\
\hline \multicolumn{5}{|l|}{ Medication at presentation, $\mathrm{n}$} \\
\hline \multicolumn{5}{|l|}{$(\%)$} \\
\hline Aspirin & $952(36.6)$ & $717(37.1)$ & $235(35.3)$ & 0.4 \\
\hline Beta-Blockers & $904(34.8)$ & $684(35.3)$ & $220(33.0)$ & 0.3 \\
\hline Statins & $932(35.8)$ & $699(36.1)$ & $233(35.0)$ & 0.6 \\
\hline ACEIs/ARBs & $1003(38.6)$ & $752(38.9)$ & $251(37.7)$ & 0.6 \\
\hline
\end{tabular}

eGFR=estimated glomerular filtration rate, $\mathrm{AMI}=$ acute myocardial infarction ,

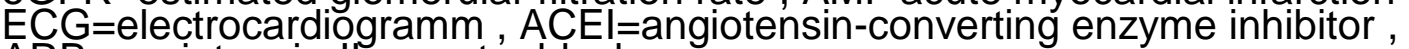
$A R B=$ angiotensin-ll receptor blocker

a Morning: 11:00 P.M - 2:00 P.M.

${ }^{b}$ Evening $=2: 00$ P.M. $-11: 00$ P.M 
Table 2A: Comparison of diagnostic accuracy for AMI as quantified by the area under the receiver-operating characteristics curve in morning and evening presenters

\begin{tabular}{|c|c|c|c|}
\hline & AUC $(95 \% \mathrm{Cl})$ & AUC $(95 \% \mathrm{Cl})$ & \\
\hline & Morninga & Evening $^{b}$ & P-value \\
\hline \multicolumn{4}{|c|}{ s-cTnl Abbott Architect } \\
\hline Oh & $0.90(0.88-0.92)$ & $0.93(0.90-0.96)$ & 0.3 \\
\hline $1 \mathrm{~h}$ & $0.94(0.92-0.95)$ & $0.97(0.93-0.99)$ & 0.3 \\
\hline $0 h+\Delta 1 h-0 h$ & $0.93(0.91-0.95)$ & $0.96(0.9-0.99)$ & 0.3 \\
\hline \multicolumn{4}{|c|}{ s-cTnl Siemens Ultra } \\
\hline $\mathrm{Oh}$ & $0.91(0.89-0.92)$ & $0.94(0.9-0.96)$ & 0.07 \\
\hline $1 \mathrm{~h}$ & $0.93(0.92-0.95)$ & $0.96(0.94-0.98)$ & 0.1 \\
\hline $0 h+\Delta 1 h-0 h$ & $0.92(0.91-0.94)$ & $0.95(0.93-0.97)$ & 0.2 \\
\hline \multicolumn{4}{|c|}{ s-cTnl Beckman Accu } \\
\hline Oh & $0.89(0.88-0.91)$ & $0.94(0.91-0.97)$ & 0.1 \\
\hline $1 \mathrm{~h}$ & $0.93(0.90-0.95)$ & $0.97(0.93-0.99)$ & 0.2 \\
\hline $0 h+\Delta 1 h-0 h$ & $0.93(0.91-0.95)$ & $0.96(0.92-0.98)$ & 0.3 \\
\hline \multicolumn{4}{|c|}{ hs-cTnl Beckman Accu } \\
\hline $\mathrm{Oh}$ & $0.91(0.89-0.93)$ & $0.94(0.91-0.97)$ & 0.3 \\
\hline $1 \mathrm{~h}$ & $0.93(0.90-0.95)$ & $0.97(0.93-0.99)$ & 0.1 \\
\hline $0 h+\Delta 1 h-0 h$ & $0.93(0.91-0.95)$ & $0.97(0.93-0.99)$ & 0.3 \\
\hline
\end{tabular}

a Morning: 11:00 P.M. - 2:00 P.M.

${ }^{b}$ Evening = 2:00 P.M. $-11: 00$ P.M.

$A \cup C=A r e a$ Under the Curve; $\Delta=$ absolute change between baseline and $1 \mathrm{~h}$ 
Table 2B: Comparison of diagnostic accuracy for AMI as quantified by the area under the receiver-operating characteristics curve in morning and evening presenters amongst early presenting patients ( $\leq 3 \mathrm{~h}$ since chest pain onset)

\begin{tabular}{|c|c|c|c|}
\hline & AUC $(95 \% \mathrm{Cl})$ & AUC $(95 \% \mathrm{Cl})$ & \\
\hline & Morning $^{\mathrm{a}}$ & Evening $^{b}$ & $P$-value \\
\hline \multicolumn{4}{|c|}{ s-cTnT Abbott Architect } \\
\hline Oh & $0.87(0.84-0.90)$ & $0.91(0.85-0.95)$ & 0.4 \\
\hline $1 \mathrm{~h}$ & $0.95(0.92-0.97)$ & $0.96(0.91-0.99)$ & 0.7 \\
\hline $0 \mathrm{~h}+\Delta 1 \mathrm{~h}-0 \mathrm{~h}$ & $0.94(0.92-0.97)$ & $0.96(0.93-0.97)$ & 0.7 \\
\hline \multicolumn{4}{|c|}{ s-cTnI Siemens Ultra } \\
\hline $\mathrm{Oh}$ & $0.89(0.87-0.91)$ & $0.95(0.92-0.97)$ & 0.02 \\
\hline $1 \mathrm{~h}$ & $0.94(0.92-0.95)$ & $0.97(0.94-0.98)$ & 0.2 \\
\hline $0 h+\Delta 1 h-0 h$ & $0.93(0.91-0.94)$ & $0.96(0.93-0.98)$ & 0.3 \\
\hline \multicolumn{4}{|c|}{ s-cTnI Beckman Accu } \\
\hline $\mathrm{Oh}$ & $0.85(0.81-0.88)$ & $0.93(0.87-0.97)$ & 0.1 \\
\hline $1 \mathrm{~h}$ & $0.94(0.91-0.96)$ & $0.97(0.91-0.99)$ & 0.5 \\
\hline $0 h+\Delta 1 h-0 h$ & $0.94(0.91-0.96)$ & $0.96(0.90-0.99)$ & 0.6 \\
\hline \multicolumn{4}{|c|}{ hs-cTnl Beckman Accu } \\
\hline $\mathrm{Oh}$ & $0.89(0.85-0.92)$ & $0.93(0.87-0.97)$ & 0.4 \\
\hline $1 \mathrm{~h}$ & $0.91(0.88-0.94)$ & $0.97(0.91-0.99)$ & 0.2 \\
\hline $0 h+\Delta 1 h-0 h$ & $0.94(0.91-0.97)$ & $0.96(0.91-0.99)$ & 0.6 \\
\hline
\end{tabular}

a Morning: 11:00 P.M. - 2:00 P.M.

${ }^{b}$ Evening = 2:00 P.M. $-11: 00$ P.M.

AUC=Area Under the Curve; $\Delta=$ absolute change between baseline and $1 \mathrm{~h}$ 


\section{Figures}

Figure 1: Median cTn concentrations at baseline in morning and evening presenters with non-cardiac chest pain, shown for all 4 assays

Figure 2: Diagnostic accuracy for acute myocardial infarction in morning and evening presenters as quantified by the area under the receiver-operating characteristic curves using four (high)-sensitive cTnl assays 